\title{
PLANES Y POBREZA EN CÓRDOBA (ARGENTINA). REFLEXIONES ACERCA DE LAS POLÍTICAS SOCIOLABORALES DESDE EL ESTUDIO DE SU IMPLEMENTACIÓN
}

\section{PLANS AND POVERTY IN CORDOBA (ARGENTINA). REFLECTIONS ABOUT SOCIO-LABOUR POLICIES FROM THE STUDY TO ITS IMPLEMENTATION}

\author{
Alicia B. Gutiérrez*
}

\section{RESUMEN}

Este artículo hace referencia a las distintas etapas por las que ha transitado la política social en Argentina, en el marco del contexto histórico en que se han desarrollado. Luego, a partir de los primeros resultados de un análisis de la implementación de dos planes en una localidad pobre de la provincia de Córdoba, Argentina, se proponen algunas reflexiones acerca de decisiones fundamentales asociadas a tres órdenes: decisiones teórico-políticas, decisiones político-instrumentales y decisiones teóricometodológicas.

PALABRAS CLAVE: ARGENTINA * POLÍTICA SOCIAL * PLANES SOCIOLABORALES * CIUDADANÍA * ESTADO * RECURSOS

\section{ABSTRACT}

This article refers to the different stages of social policies in Argentina and how they have developed within the historical context. Afterwards, from the first results of the analysis of the implementation of two plans in a poor community of Cordoba, a province of Argentina, we propose some reflections about fundamental decisions of three types: theoretical-political decisions, theoretical-instrumental decisions and theoretical-methodologic decisions.

KEYWORDS: ARGENTINA * SOCIAL POLICY * SOCIO-LABORAL PLANS * CITIZENSHIP * STATE * RESOURCES

Cátedra de Sociología (FFyH) de la Universidad Nacional de Córdoba, Argentina.

aliciagutierrez@arnet.com.ar 


\section{INTRODUCCIÓN}

En el nuevo contexto internacional, marcado por la pérdida de la centralidad del trabajo como elemento articulador de las sociedades y de la identidad de los individuos (Castel, 1997), es decir, como vínculo de filiación social, institucional, jurídica y política (Rosanvallón, 1995), la sociedad argentina (acompañada por los demás países de América Latina) sufrió un proceso de empobrecimiento general y de segmentación social, que ha sido analizado por diversos autores (Minujin, 1993a y 1993b; Murmis y Feldman, 1992; Beccaria, 1993 y Gutiérrez, 2004, entre otros). Así, la pobreza dejó de ser el producto de una crisis para constituirse en un problema estructural, resultado endémico de una nueva forma de funcionamiento económico. En ese marco, el crecimiento de las actividades con baja capacidad de absorción de mano de obra mostró la incapacidad de un modelo que desvincula crecimiento económico y desarrollo social (Benza y Calvi, 2004) ${ }^{1}$.

Las nuevas condiciones sociales a lo largo de la década de 1990, hicieron visible la situación y se incorporó la cuestión social a la agenda de gobierno: el problema del empleo adquirió una relevancia inusual, en la medida en que el aumento de la desocupación mostraba el aumento de la vulnerabilidad social.

La demanda de empleo así visibilizada y problematizada socialmente, generó nuevas modalidades de acción colectiva, como formas alternativas asociadas a diferentes tipos de prácticas: unas, tendientes a interpelar directamente a los distintos niveles del Estado, otras, más bien dirigidas a movilizar políticas de orden privado.

Entre las primeras, se observa una multiplicación de las organizaciones de base, de

1 Según datos del INDEC y de la EPH, la tasa de desocupación urbana en Argentina era en 1994 del $10,7 \%$ y llegó a un pico de $21,5 \%$, mientras el PBI se redujo en más del 11\%. En el año 2002, tras el estallido social y la devaluación de la moneda, se llegó a que el 55\% de la población viviese en hogares con ingresos inferiores a la línea de pobreza, mostrando que cada vez eran más las familias con dos generaciones de desocupados estructurales (Esquivel y Maurizio, 2005). las formas de agrupación colectiva, de acción conjunta, la creación o el fortalecimiento de antiguas redes sociales y la adopción de nuevas características en las luchas populares (Auyero, 2002a y 2002b; Svampa y Pereyra, 2003). Se produjo entonces el desplazamiento del conflicto laboral desde el área industrial al sector público, generando un problema para los representantes del Estado en sus diferentes niveles, municipal, provincial y nacional, quienes tenían que responder por los conflictos en la relación capital-trabajo. Las modalidades de la protesta social cambiaron en esta década: tomas y ataques a edificios públicos (casas de gobierno provinciales, legislaturas, sedes de gobiernos municipales, tribunales, etc.), cortes de rutas nacionales y provinciales, los campamentos en plazas centrales se generalizaron $y$ aumentaron su frecuencia en distintas zonas del país (Auyero, 2002a).

Entre el segundo tipo de prácticas, se destacan las ligadas a los movimientos de trabajadores auto-convocados, las ferias de trueque, las fábricas recuperadas, los comedores, las salitas de salud, las panaderías comunitarias, etc. Además, ciertas organizaciones populares establecieron lazos con ONGs y fundaciones, tendiendo a articularse en redes con otras organizaciones comunitarias no estatales. Se trata de un "modelo de acción territorial" (Svampa y Pereyra, 2003) que responde a las nuevas formas de auto-organización de lo social, desvinculadas de la acción sindical tradicional. Aquí también es visible cómo el conflicto del trabajo se traslada desde la fábrica o unidad de producción, al barrio y a la calle, al espacio público.

Frente a este escenario, se imponía tomar medidas urgentes con el fin de aliviar las durísimas condiciones de la población, disminuir la conflictividad social y de ese modo, asegurar la "gobernabilidad". En un primer momento, se intentó paliar la situación utilizando el recurso de poder de la coerción, a partir de la represión de las manifestaciones y cortes de ruta por parte de la Gendarmería Nacional. Sin embargo, esas medidas no dieron el resultado esperado, desde el momento en que los conflictos se multiplicaban en diversos puntos del país. Es entonces cuando comienzan 
a diseñarse nuevos planes sociales focalizados sobre la población desempleada, como mecanismos de control estatal sobre los sectores populares movilizados.

La crisis económica, social y política de diciembre de 2001, mostró con crudeza la realidad social argentina que había empezado a construirse a mediados de la década de 1970. Sin embargo, a partir de 2003, con la nueva orientación política y económica que se adopta desde el gobierno nacional, comenzaron a visualizarse otras estrategias para hacer frente al problema estructural del desempleo y de la pobreza.

Al tomar en cuenta la breve caracterización de este contexto, se hará referencia, en primer lugar, a las distintas etapas por las que ha transitado la política social en Argentina; luego, se presentarán los primeros resultados de un análisis de la implementación de dos planes en una localidad pobre de la provincia de Córdoba, Argentina, para finalmente, proponer algunas reflexiones en torno a esta problemática.

\section{LA POLÍTICA SOCIAL EN ARGENTINA: TRABAJO-CIUDADANÍA, "DES-CIUDADANIZACIÓN", "RE-CIUDADANIZACIÓN"}

Brevemente, puede decirse que la política social argentina ha transitado por un proceso que ha tendido desde la integración-universalización de derechos, hacia la exclusión asistencializada que está implicada en las políticas focalizadas. El trabajo asumió un lugar central en la reproducción de la vida social y en su articulación con el desarrollo de la política social. En otras palabras, el trabajo fue la base y la condición de los derechos ciudadanos, en épocas de pleno empleo (Hintze, 2006).

La presencia del Estado de Bienestar en la Argentina y su contenido específico, ha sido objeto de discusión (Minujin, 1993c; Bustelo, 1988 y 1993; Barbeito y Lo Vuolo, 1995; Grassi, Hintze y Neufeld, 1994; Hintze, 2006). Más allá de debates específicos, puede decirse que sus principales características son:

a) se desarrolló en Argentina antes y de manera más amplia que en el resto de América Latina; b) su modalidad estaba demasiado ligada a la figura del trabajador (más que a la del ciudadano), hecho potenciado por la relación que existía entre el Estado y los sindicatos ${ }^{2}$;

c) en consecuencia, su instauración fue parcial en Argentina, dejando importantes áreas sociales y grupos de población sin cubrir: aquellos que por razones diversas no pudieran incorporarse al mercado de trabajo (Grassi, Hintze y Neufeld, 1994).

Simultáneamente con el proceso de empobrecimiento al que se ha hecho referencia anteriormente, se fue avanzando por un camino de pérdida de derechos, respecto a la relativa universalidad precedente. Así, de la crisis del Estado de Bienestar y sus intentos de reforma se pasó a la producción del "Estado de Malestar" (Bustelo, 1993).

Muchos otros autores han continuado profundizando estas cuestiones (Lo Vuolo, 1995; Guimenez y Zibecchi, 2005; Grassi, 2003 y 2004; Hintze, 2006, por nombrar solo a algunos), que apuntan a aspectos diferentes de la problemática. Aquí, vale la pena subrayar ciertos rasgos simbólico-políticos asociados a las modalidades de la política social de esta etapa:

a) Se pasa de una intencionalidad universalista e igualitaria a un asistencialismo compensatorio, en relación con dos momentos diferentes de la década de 1990; en la primera mitad, las políticas focalizadas se orientaron a la atención de la pobreza (a grupos biológicamente vulnerables: tratamiento de la desnutrición, asistencia a madres $y$ niños, etc.); en la segunda mitad, los planes de empleo se presentaron para responder al acuciante problema de la desocupación, bajo distintas modalidades (subsidios directos a desocupados, sujetos a capacitación

2 Por esto mismo, la universalización ligada a los derechos de ciudadanía, más que un principio, fue un resultado de un contexto de pleno empleo, dada la amplitud de la categoría "trabajador", casi superpuesta a la de ciudadano (Hintze, 2006). 
o contraprestación laboral, creación de empleo público temporal — pasantíasy subsidios a la creación de empleo en el sector privado) (Hintze, 2006).

b) Todas estas prácticas tienen consecuencias importantes en el nivel de las estrategias de las familias inmersas en la pobreza: los beneficiarios de estas políticas se construyen a sí mismos, no desde sus potencialidades (capitales objetivos e incorporados), sino desde el lugar del "sujeto carenciado". Es decir, dominan ampliamente las apuestas realizadas desde las falencias que habilitan a conseguir diferentes tipos de ayudas estatales, que las sustentadas en las capacidades y sobre todo, en los derechos a exigir, individual y colectivamente, la inserción plena en la sociedad. Estos procesos cobran dimensiones mayores cuando se encuentran relacionados con prácticas clientelares, como lo han mostrado diversos autores (Auyero, 2001 y 2002; Svampa, 2005; Lodola, 2005).

c) Es posible observar también modificaciones en otro nivel de las estrategias; claramente, esta dinámica de la ciudadanía asistida-focalizada (Svampa, 2005) produce transformaciones en el modo de relacionarse las bases entre sí, fundamentalmente por el quiebre de los vínculos y de los lazos solidarios entre pares, $y$ entre esas bases y el poder político, mostrando con mayor crudeza las relaciones de dominación.

d) En consecuencia, como señala Grassi, durante la década de 1990, en Argentina, "la política se empobreció, al quedar reducida a las pujas de corto plazo, en la misma medida en que se empobreció la economía al quedar licuada en las reglas del mercado libre y se despreció la "reflexividad social crítica", encerrada en la racionalidad técnica, que expropió para ese limitado campo los temas que hacen a las metas deseables y elegibles por la sociedad" (2003b:162, entrecomillado de la autora).
Ahora bien, siguiendo a Hintze (2006), es necesario subrayar que al producirse la mencionada crisis económica, social y política de 2001, emergió un nuevo contexto para re-pensar los derechos y la política social. Se hace visible entonces otra cara del proceso social vivido en la Argentina de la década anterior, que genera aportes para la reconstrucción de esa "reflexividad social crítica", cuya ausencia había contribuido al "empobrecimiento" de la política. De este modo, asambleístas, movimientos de trabajadores desocupados, organizaciones sindicales alternativas, movimientos de empresas recuperadas y grupos de la cultura, fueron elaborando un discurso reactivo frente al modelo neoliberal, pero también construyeron las bases de propuestas de acción tendientes a recuperar formas de democracia participativa y de autonomía socioeconómica. Surge así la idea de una ciudadanía comprometida, distinta del concepto de ciudadanía propia del liberalismo, que exige moralmente poco: respetar las leyes y pagar los impuestos (Hintze, 2006).

Frente a los discursos de focalización como garantía de equidad, se recupera entonces la universalización de derechos y a partir de 2003, comienzan a implementarse programas que intentan promover formas asociativas $y$ el trabajo autogestivo, propuestos a nivel discursivo como una reversión de la política social anterior (Hintze, op. cit.).

Es decir, desde el discurso oficial y desde la formulación de una nueva política social, se puede decir que se intenta transitar desde un proceso de "des-ciudadanización" a uno de "reciudadanización", como se intentará mostrar en los apartados siguientes.

\section{PLANES SOCIALES EN UNA LOCALIDAD POBRE DE LA PROVINCIA DE CÓRDOBA, ARGENTINA}

En el marco de una investigación en curso acerca de redes sociales en la pobreza ${ }^{3}$,

3 Se trata de una investigación acerca de la producción y reproducción de la pobreza, a partir del análisis de redes sociales, que se enlaza con un conjunto de proyectos individuales. El análisis preliminar del PJJHD se realizó sobre la base de un relevamiento general de la Tercera Sección 
que se está realizando en Malvinas Argentinas (provincia de Córdoba, Argentina), se presentarán los primeros resultados que arrojan los análisis realizados en torno a la implementación de dos planes: Plan "Jefas y Jefes de Hogar Desocupados" (PJJHD) y Plan "Manos a la obra", puestos en marcha en esa localidad.

Malvinas Argentinas está ubicada a 16 km de la ciudad de Córdoba, capital de la provincia del mismo nombre. Nació como un caserío a la vera del ferrocarril en el siglo XIX, pero fue en las dos últimas décadas del siglo $\mathrm{Xx}$ cuando cobró impulso la radicación de la población y se observó una marcada reactivación en la venta de lotes.

Se trata de una localidad que presenta graves deficiencias en infraestructura y equipamientos urbanos, que carece de dinámica económica propia y es altamente dependiente de la ciudad de Córdoba. Al mismo tiempo, ofrece ventajas para las condiciones de vida de sus habitantes, principalmente por su cercanía a la capital (centro de abastecimiento y de trabajo) y por el costo accesible del suelo y de la vivienda. Por todo ello, se constituyó en un municipio receptor de población de menores ingresos, fundamentalmente, provenientes de la ciudad de Córdoba, cobrando así características de "ciudad dormitorio de pobres" (Tecco y Bressan, 2003).

Según datos del Censo 2001, Malvinas Argentinas contaba con una población de 8628 habitantes, un nivel que es un $67 \%$ más alto que el del Censo 1991 (5160 habitantes).

En el año 2004, tenía un total de 2756 viviendas ocupadas, con un promedio de 4,5 personas por vivienda (para la provincia y la nación este promedio alcanzaba respectivamen-

Malvinas Argentinas (la que concentra los mayores índices de pobreza), llevado adelante por el equipo de investigación durante los meses de febrero y julio de 2007. La encuesta se aplicó sobre un $n$ total de 186 casos (tomando a la familia como unidad de análisis), que representan aproximadamente el $90 \%$ de los residentes de esa sección. La información sistematizada y analizada hasta el momento del Plan Manos a la Obra, en cambio, fue obtenida a partir de entrevistas con informantes claves (la encargada por el municipio de la implementación del PMO en la localidad y microemprendedores) y de observación. te al 3,4 y 3,6). Además, en ese año trabajaban 1,5 personas por hogar, mientras el ingreso mensual medio por hogar ascendía a $\$ 514$.

Del $68 \%$ de población sin NBI (Necesidades Básicas Insatisfechas) según datos del Censo 2001, se pasa a un 60\% en el 2004, como así también del 75\% de hogares sin NBI se desciende al 68\%; es decir, se observa un empeoramiento de las condiciones generales de alrededor de 8 puntos en el breve lapso de tres años.

Según datos de la Secretaría de Desarrollo Social de la localidad, en el año 2008, se distribuían a nivel municipal como gestor de planes nacionales, 50 PJJHD, 456 Plan Familias, 9 PEC (Programa de Empleo Comunitario) y 5 proyectos "Manos a la obra". Además, existen familias que reciben planes sociales de origen provincial que se distribuyen a través del Ministerio de Desarrollo Social de la Provincia de Córdoba: 17 Programas "Familia Joven" y otros (Programas "Igualdad de Oportunidades", "Trabajo para todos" y "Volver al trabajo").

\subsection{EL PJJHD EN MALVINAS ARGENTINAS EN EL MARCO DE EVALUACIONES GLOBALES}

Como consecuencia de la crisis social, política y económica de diciembre de 2001 y ante el recrudecimiento de la situación crítica del desempleo, desde el Estado nacional se pone en marcha el Plan Jefes y Jefas de Hogar Desocupados (PJJHD) ${ }^{4}$, que a mediados de 2003 ,

4 Este plan social comienza a implementarse a principios de 2002 y "alcanzó el millón de hogares en mayo y cuatro meses después superaba la cifra de 1,8 millones; el pico se registró hacia mediados de 2003, con cerca de dos millones. Se constituyó, entonces, en el programa de asistencia más grande que se haya puesto en funcionamiento en Argentina $y$ sin duda, se ubica entre los de mayor cobertura en la región. El monto erogado en 2002 fue de $\$ 760$ millones, trepando a más de $\$ 3000$ el año siguiente. Estas cifras representaron el 1,6\% $y$ el $5,2 \%$, respectivamente, del conjunto del gasto del sector público federal, $2,4 \%$ y $8,0 \%$ del gasto social nacional y el 1,2\% y 4,3\% del gasto consolidado social" (Beccaria, 2006: 7). El PJJHD se constituyó en la acción de emergencia más importante de la época en materia de política de empleo y sigue vigente en la actualidad. A través de este plan se otorgaba al beneficiario un subsidio de $\$ 150$ mensuales, según ciertos requisitos de elegibilidad: ser 
llega a aproximadamente a 2000000 de beneficiarios y logra modificar los índices de desocupación.

Iniciado a principios de 2002, este plan se constituyó en un ingreso de subsistencia para muchas familias (Giacometti y Monza, 2003) y fue un ejemplo paradigmático de la ayuda social, aunque sufrió algunas transformaciones en su implementación y características. Luego, se sumaron otros planes y programas, tanto a nivel nacional como provincial ${ }^{5}$.

En Malvinas Argentinas, se han observado situaciones coincidentes con los análisis realizados a nivel nacional, según las siguientes características de los beneficiarios de PJJHD:

a) Tamaño y composición del hogar: en esta localidad hay un mayor tamaño promedio de los hogares que son beneficiarios del Plan (5,52 miembros); esto es así, tanto si los comparamos con aquellos hogares encuestados que no son beneficiarios de una ayuda social (4,46 miembros) como así también, si tomamos de referencia la media de personas por hogar que se desprende del censo provincial del año 2004 (4,5 miembros). La misma tendencia se observa en relación

jefe o jefa de hogar, estar desocupado(a) al momento de recibir la prestación y tener al menos un hijo menor de 18 años viviendo en el hogar. A cambio se exigía una contraprestación que podía ser laboral o no (asistir a actividades de capacitación) además, garantizar que los hijos del hogar asistieran a la escuela y cumplieran con ciertos controles de salud.

Dada la cantidad inusual de beneficiarios del PJJHD y las dificultades para garantizar el cumplimiento de los requisitos que este establecía (entre otros, ofrecer una contraprestación laboral al beneficiario) en el año 2005, se dispone el pasaje de algunos de sus beneficiarios hacia el Plan Nacional Familias. En la provincia de Córdoba, a partir de 2007 y bajo el gobierno de Schiaretti, se implementan planes sociales de empleo de origen provincial que se distribuyen a través del Ministerio de Desarrollo Social de la Provincia de Córdoba: Programas "Familia Joven", "Igualdad de Oportunidades" y "Trabajo para todos", que se suman al programa "Volver al trabajo", puesto en marcha en julio de 2000, bajo el gobierno de De la Sota $y$ vigente hasta diciembre de 2007. con la presencia de menores: 0,92 frente a 0,56 para la presencia de menores de 5 años y 3,06 frente a 1,68 para menores entre 6 y 17 años.

Ello es coincidente con análisis globales respecto a la implementación del Plan, donde se destaca el mayor tamaño promedio de los hogares con beneficiarios, vinculado a la situación de pobreza y a una mayor presencia de menores (representan más del 44\% del total de miembros, 20 puntos porcentuales más que en los hogares sin beneficiarios) (Pautassi, 2003).

b) Tasa de dependencia: de los análisis de la implementación del PJjHD, en general, se desprende también que dado el mayor tamaño de los hogares pobres y la importante presencia de menores de edad, la tasa de dependencia en ellos suele ser más elevada. Esto se confirma para los hogares encuestados en Malvinas Argentinas, donde los hogares que reciben el Plan presentan un índice de 0,33 frente al de 0,45 de los hogares no-beneficiarios.

c) Ingresos del hogar: a nivel global, el impacto del PJJHD sobre el nivel de ingreso de los hogares presenta una serie de características. En general, puede decirse que su incidencia en los niveles de pobreza no es alta; el 93\% de los hogares que percibe el beneficio continúa bajo la línea de pobreza. Sin embargo, el acceso al Plan ha disminuido en $4 \%$ la incidencia de la pobreza en los hogares beneficiarios. Por otra parte, sus efectos son más notorios en los niveles de indigencia: "en promedio, uno de cada cinco hogares que accedió al programa habría estado en una situación de indigencia sin éste" (Pautassi, 2003: 96). Esto coincide con observaciones realizadas desde otras perspectivas, que plantean que "el plan jefes tuvo una cobertura restringida. El 24,5\% de los hogares pobres y el 35,5\% de los hogares indigentes eran beneficiarios; quedaban por lo 
tanto sin cubrir el $75 \%$ de los pobres y el 64,5\% de los indigentes" (Cortés et ál., 2004: 17 y 19).

En Malvinas Argentinas, es posible observar que los hogares beneficiarios del Plan son aquellos cuyos ingresos promedio sin la ayuda social son un $40 \%$ menor que el de los hogares que no reciben ningún tipo de ayuda estatal $(\$ 1012,93$ frente a $\$ 1211,18)$. Asimismo, es importante subrayar que a pesar de que los hogares beneficiarios son aquellos que están en peor situación comparativa en términos de ingresos, del análisis de los ingresos per cápita de los integrantes del hogar y de la información provista por el Instituto Nacional de Estadística y Censos del país, puede concluirse que tanto los miembros de hogares beneficiarios como aquellos sin ayuda estatal, se hallan por debajo de la línea de pobreza establecida a nivel nacional para la fecha en que fue tomada la encuesta. Sin embargo, los integrantes de hogares sin PJjHD se encuentran más distantes de la Línea de Indigencia, mientras que los miembros de hogares beneficiarios se hallan al límite de esta, mostrándose aún más vulnerables que los primeros en este sentido ( $\$ 308,53$ de ingreso per cápita para hogares que no reciben Plan, frente a $\$ 197,72$ para hogares beneficiarios). Así, el aporte de $\$ 150$ que ingresan al hogar a partir del Programa, resulta importante para que una proporción significativa de los hogares beneficiarios situados en niveles de pobreza puedan mantenerse en esa posición y no caer en la indigencia ${ }^{6}$.

En coincidencia con los resultados obtenidos para la localidad de Malvinas Argentinas, también desde otras perspectivas se hace hincapié en señalar la magra capacidad del Plan para intervenir en solucionar la situación de pobreza de sus beneficiarios, dada:

... la escasez de las transferencias y los beneficios frente a una situación altamente crítica, y con la distribución de los derechos de acceso al programa, ya

$6 \quad$ En efecto, para febrero de 2007, la línea de pobreza se fijaba en 298,27 y la de indigencia en 138,73; para julio de 2007, la primera era de 300,95 y la segunda de 139,98 por adulto equivalente. que la historia ocupacional de los beneficiarios revela que los más vulnerables, el núcleo duro de la desocupación y el desaliento, no estuvieron representados adecuadamente (Cortés et ál., 2004: 27).

d) Nivel educativo de los miembros del hogar: a partir de la información revelada en la encuesta de Malvinas Argentinas, es posible realizar un análisis de las frecuencias acumuladas en función del máximo nivel de estudios alcanzado por al menos un miembro del hogar. Así, se puede observar que la mayor parte de la población encuestada se distribuye entre las categorías: Primaria Completa, Secundaria Incompleta y Secundaria Completa. Sin embargo, se destaca la existencia de una diferencia significativa en términos de logros educativos, a favor de aquellos hogares que no reciben el PJJHD, puesto que en este grupo el porcentaje de quienes han logrado culminar el nivel medio duplica al de aquellos integrantes de hogares beneficiarios que finalizaron el secundario: $29,2 \%$ frente a $14,1 \%$. Es decir, los beneficiarios del PJJHD son hogares cuyos integrantes han alcanzado en promedio un nivel educativo más bajo.

e) Trayectorias laborales de los beneficiarios y precarización en la inserción laboral: varios análisis hacen hincapié en destacar la trayectoria laboral de los beneficiarios, en tanto este resulta un indicador relevante del impacto del plan en términos de focalización de la ayuda social. Así, en general, se observa que:

... los beneficiarios de los planes exhiben trayectorias económicas diferenciadas según género y posición en el hogar. Entre los varones predominan las trayectorias más vulnerables pero entre los jefes de hogar son mayoritarias las trayectorias de siempre ocupados, y en segundo término las trayectorias de inserción intermitente. Entre las cónyuges predominan sobre todo las inserciones de inactividad $y$ desaliento (Cortés et ál., 2004: 24). 
Ahora bien, en Malvinas Argentinas, los jefes de los hogares beneficiarios se han desempeñado en categorías ocupacionales asociadas al cuentapropismo. Dadas las características del mercado laboral argentino, se puede sostener que este es un indicador de una trayectoria laboral desarrollada en un contexto de mayor vulnerabilidad y precarización: en efecto, en los sectores pobres el trabajo por cuenta propia está ligado a una mayor inestabilidad en las fuentes de empleo y a la carencia en la cobertura de salud y previsión social.

En conclusión y al igual que lo observado a nivel nacional, la implementación del PJJHD en Malvinas Argentinas, constituyó un mecanismo de estabilización de ingresos (ya sea ante la ausencia de ingresos o complementando ingresos) de los hogares con inserciones laborales precarias - de baja remuneración- o con trayectorias intermitentes (Cortés et ál., op. cit.), impidió - o al menos limitó - la caída de una buena parte de la población en la indigencia $y$ dadas las características de sus beneficiarios, puede decirse que cumplió con sus objetivos de focalización.

De este modo, el PJJHD es un claro ejemplo de las estrategias estatales frente a la cuestión del desempleo, partiendo de un recorte de la población a la que está dirigida la política social: aquellas personas desocupadas, jefes de familia con hijos a cargo menores de 18 años. Esta estrategia lleva implícita, indudablemente, una determinada concepción acerca de los derechos $y$ acerca de quiénes pueden tener acceso a ellos, generando consecuencias para la construcción de un nuevo modelo de ciudadanía. En efecto, el esquema de la aplicación de políticas sociales focalizadas supone que el derecho de los ciudadanos a recibir la protección del Estado está sujeto a la condición de los mismos en función de su carácter de desempleados, de su nivel de ingresos, de la cantidad de hijos, etc. $y$ no a su condición de sujetos miembros de un colectivo social.

\subsection{PLAN NACIONAL DE DESARROLLO LOCAL Y ECONOMÍA SOCIAL "MANOS A LA OBRA" (PNMO)}

Siguiendo a Hintze (2006), este plan es la política que más se aleja del modelo anterior. El discurso oficial asocia al modelo liberal, a sus políticas públicas y a las nefastas consecuencias en la sociedad argentina, un conjunto de características de las cuales aquí se destacan dos: el retiro de la intervención del Estado y un proceso de "desciudadanización" (A. Kirchner, cit. por Hintze, op. cit.).

Frente a ello, propone, a la inversa, "trabajar desde una política social integral" (A. Kirchner, cit. por Hintze, op. cit.) que supone una vuelta a la intervención del Estado y lo que se podría llamar, la generación de un nuevo proceso de "ciudadanización" o una nueva "re-ciudadanización", fundamentado en "la promoción de oportunidades" y "el fortalecimiento del capital social" (A. Kirchner, cit. por Hintze, op. cit.).

En efecto, el PNMO se lanzó a nivel nacional en agosto de 2003 y llegó a la provincia de Córdoba entre fines de ese año y comienzos de 2004. Desde la Municipalidad de Malvinas Argentinas, se presentaron numerosos proyectos, pero funcionaron finalmente cinco emprendimientos: uno destinado a fabricar ladrillos y bloques, una sodería, una marroquinería, un emprendimiento ligado a la cría de conejos y otro destinado a la confección de manualidades y costuras.

En su implementación, el PNMO presentaba dos grandes brazos:

1) Una línea de subsidios destinados a los titulares de un PJJHD. En este marco, sus beneficiarios seguían cobrando los $\$ 150$ correspondientes a este plan, pero podían a su vez presentar proyectos productivos para ser financiados por el PNMO. Dentro de esta línea, se entregaban hasta $\$ 5000$ para una persona, $\$ 10000$ para dos y $\$ 15000$ como monto máximo para 3 o más.

El proyecto elaborado debía pasar una revisión realizada por el Consejo Consultivo, quien lo evaluaba y aceptaba, junto con el intendente. Si el proyecto era aprobado se lo elevaba a la Nación. Los Consejos Consultivos se constituían por iniciativa del municipio y estaban integrados por el intendente, concejales $y$ actores representativos de la localidad. 
2) Otra línea de subsidios estaba destinada a emprendimientos asociativos productivos para 2, 3 o más personas. Dentro de esta línea del programa, el procedimiento consistía en elaborar un borrador con el proyecto de emprendimiento con los interesados, en el que una persona contratada por la municipalidad actuaba como asesora en el armado del cuestionario y la ficha, daba respuestas a dudas que pudieran plantearse $y$ luego, se entregaban a la Secretaría de Acción Social. Posteriormente, una ONG lo controlaba y citaba a los emprendedores si encontraban algún aspecto que era necesario revisar para poder hacer la redacción final del proyecto ${ }^{7}$. En esta línea de subsidios se otorgaban, por un lado, hasta \$15000 para la compra de herramientas $y$ maquinarias $y$ por otro lado, dinero para la compra de materias primas.

Para la primera línea de subsidios, la convocatoria fue masiva: se atendieron entre 600 y 700 interesados. Para la segunda, se otorgó asesoramiento para alrededor de 30 proyectos, aunque la mayoría de las solicitudes aprobadas corresponden a esta segunda línea.

Según la encargada de asesorar y de recibir a los emprendedores, "en general la evaluación del plan es positiva", destacando fundamentalmente tres cuestiones:

a) El Municipio designó a una persona cuya tarea específica era acompañar a los interesados en todo el proceso de formulación y entrega de los proyectos.

b) El Municipio actuó como organismo de transferencia del dinero $y$ de control de rendición de gastos, proceso luego auditado por el gobierno nacional.

c) El otorgamiento de un subsidio (no un crédito), lo que implicaba la devolución del $10 \%$ del total, con transferencias del producto que se obtenía y que era recibido

$7 \quad$ Dentro de lo estipulado en el PNMO, había una línea de financiamiento para las ONG que se dedicaran a capacitar y asesorar a emprendedores. por una institución de la localidad que lo necesitara.

Entre los aspectos negativos, para ambas líneas de subsidios, se señalan:

a) La extensión de los formularios, la cantidad y complejidad de los requisitos. Estas dificultades eran en parte subsanadas con el asesoramiento del Municipio, pero de alguna manera:

... desalentaban mucho a la gente, que no creían que luego podían ser capaces de llevar adelante el emprendimiento; algunos venían muy entusiasmados pero luego no volvían más (...). Una de las cosas que más espantó fue el armado del proyecto. Poder bajar las ideas y escribirlas en los formularios. Organizar la empresa y los trabajadores, los horarios, las tareas, etc. (Lic. Lía Soti, Secretaría de Acción Social, Municipalidad de Malvinas Argentinas).

b) La demora en la evaluación, en la entrega de los proyectos y en la transferencia del financiamiento: "Yo me desesperaba con las demoras, porque veía la necesidad de la gente (...). Incluso todavía hoy viene gente preguntando ¿cuándo van a salir los subsidios?" (Lic. Lía Soti, Secretaría de Acción Social, Municipalidad de Malvinas Argentinas).

c) Ausencia de mecanismos que aseguraran un seguimiento en el tiempo de los emprendimientos.

d) Fundamentalmente, falta de capacitación para los emprendedores. En este aspecto, tomando el discurso de los entrevistados, podemos diferenciar tres tipos de capacitación: "capacitación técnica en el rubro", "capacitación empresaria" y "capacitación motivacional".

Respecto a la primera, las dificultades se asocian concretamente con el capital cultural incorporado por los interesados, que implicaban situaciones diferenciales para quienes pretendían participar en las dos líneas previstas 
por el Plan. Un caso era el de los microemprendimientos asociativos, que contaban con una suerte de capital familiar incorporado:

En general los emprendimientos comenzaban desde cero, prácticamente el 95\%; los comenzaron a hacer como podían, tenían buena clientela y capacitación en su oficio: por ejemplo había gente que habían sido trabajadores de fábrica, o el padre había sido carpintero, había conocimientos del rubro en general" (Lic. Lía Soti, Secretaría de Acción Social, Municipalidad de Malvinas Argentinas).

Para el caso de los JJHD, la situación era todavía más compleja:

El caso para los PJjHD fue más difícil, puesto que el nivel educativo y el nivel de capacitación de sus beneficiarios en esta población era bajo o muy bajo, incluso primario incompleto (...). Primero, que la mayoría eran mujeres que no habían trabajado nunca, $y$ muchos de los hombres tenían una historia de desocupación, no se habían capacitado en ningún oficio particular (Lic. Lía Soti, Secretaría de Acción Social, Municipalidad de Malvinas Argentinas).

Respecto a la "capacitación empresaria", se puede decir que era una necesidad que estaba ligada a la formulación e implementación de los proyectos de las dos líneas del Plan. Primero, se exigía una suerte de "ejercicio de mentalidad empresaria" como por ejemplo, poder realizar un cálculo de la productividad estimada por seis meses o poner en marcha disposiciones fundamentales para hacer que los emprendimientos funcionaran, tales como, la necesidad de que parte de las ganancias fueran reinvertidas y no destinas en su totalidad a las urgencias diarias propias de las condiciones de vida de los sectores pobres. Esa "mentalidad empresaria" exigía también conocimientos básicos de comercialización, de marketing, de exploración mínima del mercado, etc. En resumen, esta capacitación implicaba poner en tensión "disposiciones empresariales" frente a las "disposiciones del manejo de las economías domésticas". En otras palabras, se trataba de generar disposiciones que se adecuaran a las nuevas condiciones objetivas, frente a las que históricamente vienen incorporando quienes viven en condiciones de pobreza $y$ de inseguridad cotidiana. Son disposiciones respecto al tiempo y a las posibilidades de espera de resultados, de disposiciones que permitan percibir y distinguir el corto, el mediano y el largo plazo (generalmente, ajenas a quienes han incorporado una historia objetiva donde prima la urgencia y "el día a día"), de disposiciones respecto a prácticas y representaciones que impliquen una separación del patrimonio personal y el del emprendimiento, respecto a la toma de decisiones de gastos, de reinversiones, etc.

Finalmente, "la capacitación a nivel motivacional" es considerada como necesaria para poder canalizar las expectativas relativas a la iniciativa de "hacer algo" y no estar en condiciones de sugerir qué actividad podía ser posible, a partir de sus posibilidades concretas de conocimiento del rubro, de condiciones objetivas de ofrecer un mínimo de los requisitos solicitados (locales, oportunidades de adecuarlos a las exigencias de salubridad $y$ de la puesta en marcha del microemprendimiento, etc.).

Muchas personas se acercaban y me decían:-Quiero hacer algo, ¿pero qué puedo hacer? Y yo les respondía: -a ver, dígame, ¿usted qué sabe hacer? (Lic. Lía Soti, Secretaría de Acción Social, Municipalidad de Malvinas Argentinas).

—_El Plan Manos a la Obra? La verdad es que no sé qué es. Nosotros con mi marido teníamos necesidades y nos enteramos de que el Intendente daba subsidios. Fuimos y no sabíamos bien qué hacer. Al final, como él tenía experiencia decidimos un taller de microfundición (Mirtha, emprendedora).

En el año 2005, hubo evaluaciones acerca del funcionamiento del PNMO y se tomó la decisión de no financiar más emprendimientos aislados, sino de intentar establecer cadenas 
productivas que fortalecieran el desarrollo local. En Malvinas Argentinas, desde el municipio y la ONG SEHAS se propuso un proyecto complejo para tres años, que articulaba emprendimientos de comida, costura y construcción, intentando promover, a la vez, cuestiones relativas a problemáticas de género $y$ de ciudadanía que fue elevado al Estado nacional y por el cual no hubo respuesta.

\section{REFLEXIONES FINALES}

Para comenzar a proponer algunas reflexiones finales, se hace una recopilación de lo planteado hasta el momento en este artículo. Primero, un contexto histórico signado por un fuerte proceso de empobrecimiento $y$ de segmentación social, en el marco de un modelo económico que hace visible su cara más perversa con la crisis económica, social y política de diciembre de 2001. Segundo, una política social que ha pasado por diferentes etapas que implican distintas nociones de "ciudadanía": desde una asociada a la noción de trabajador a una etapa que ha sido llamada "ciudadanía asistida-focalizada" (Svampa, 2005), atravesando por un proceso que va desde una "des-ciudadanización" hacia una "re-ciudadanización". Tercero, el análisis de los primeros resultados de la implementación de dos planes sociales en una localidad pobre: el primero, como parte de una estrategia más bien impulsada por las urgencias de la "gobernabilidad" y que pone en juego una "ciudadanía asistida"; el segundo, marcado más claramente por una política tendiente a la "re-ciudadanización", pero que también muestra sus límites.

¿Cómo podría avanzarse en ese proceso que he llamado de "re-ciudadanización"? O de otro modo, ¿Cómo contribuir a hacer que los derechos sociales sean efectivos y accesibles para todos? ¿Cómo intentar realmente que las oportunidades sociales no queden definidas solo en el plano teórico?

Se podría empezar diciendo que es necesario tomar decisiones frente, al menos, tres cuestiones:

a) Decisiones teórico-políticas fundamentales, tomando al campo de la política como instancia de constitución del orden social, en el sentido de Grassi, como:
... el espacio de la compleja configuración del poder social, constituido por los campos donde se procesan luchas y disputas por la orientación del sentido y por la dirección de los acontecimientos, en cuyo devenir también se constituyen los sujetos que expresan, así, la voluntad de imponer una "normatividad" y orientar la "normalización" de un orden político (2003a: 304, entrecomillado de la autora).

Evidentemente, ciertas apuestas de esas luchas giran en torno a ciertas preguntas centrales ¿qué papel cumple el Estado?, ¿cuál es el que tendría que cumplir?, ¿cuál es el que ha venido cumpliendo históricamente? Decisiones de este orden, como se ha mencionado brevemente, han sido tomadas en nuestro país por la nueva orientación política que se ha impuesto desde 2003.

Ahora bien, ¿cómo se asegura el efectivo ejercicio de los derechos ciudadanos?

Es necesario tener en cuenta dos aspectos, que de algún modo, constituyen supuestos teóricos fundamentales. En primer lugar, implica distinguir, analíticamente, el plano de los "derechos formales" y el de los "derechos sustantivos". Más precisamente, conceptualizar al "ciudadano" no solo en su condición de parte de una comunidad, con derechos y obligaciones formalmente reconocidos a todos, sino también, como agente social cuyas capacidades de ejercicio de esos derechos están asociadas a la disponibilidad de los recursos objetivos e incorporados que posee, según las relaciones sociales que estructuran esa "comunidad".

En segundo lugar, que el plano de la política constituye un campo de luchas, simbólicas por excelencia, donde las decisiones no surgen de un consenso espontáneo, sino de acuerdos y negociaciones que se establecen entre agentes e instituciones que se insertan en relaciones de fuerza.

Solo reconociendo estos dos aspectos es que se puede plantear, como apuesta política fundamental, una verdadera universalización de derechos. Es decir, fijar la meta de asegurar 
a todos el ejercicio pleno de los derechos formales, según el plano de los derechos sustantivos. Ello supone, claramente, el rol activo de un Estado que en representación del colectivo pueda, con su fuerza objetiva y simbólica, defender a quienes ocupan las posiciones dominadas del espacio.

b) Decisiones político-instrumentales, necesarias para operacionalizar las grandes decisiones tomadas anteriormente, tendientes a responder preguntas del tipo: ¿cuáles son las instituciones que pueden asegurar el acceso a los diferentes tipos de bienes a los que ocupan las posiciones dominadas del espacio social?, ¿de qué modo se pueden potenciar su participación y su gestión?, ¿qué otros mecanismos pueden visualizarse como viables?, ¿qué tipo de políticas redistributivas permitirían con mayor eficacia reducir las distancias sociales?, etc.

En un marco colectivo que implica siempre relaciones de fuerzas, derivadas de la distribución desigual de los recursos y teniendo como meta la universalización de los derechos que realmente consoliden un proceso de "reciudadanización", será necesario, en líneas generales, tomar decisiones que promuevan las formas asociativas y el trabajo autogestivo, tal como se ha mencionado anteriormente.

Esto significa, de algún modo, la necesidad del fortalecimiento del "capital social", por otra parte, como se ha visto, explícitamente sostenido en el discurso del gobierno nacional.

c) Decisiones teórico-metodológicas que sustenten los análisis.

Indudablemente, las decisiones de las dos órdenes anteriormente mencionadas, necesitan estar sustentados en investigaciones concretas de la realidad social, sobre las cuales se pretende intervenir. La cuestión es amplia y compleja. Acá se va a hacer referencia a dos aspectos: el concepto de capital social implicado en las propuestas y las condiciones objetivas de los destinatarios de la política social.

Más allá de los distintos debates que ha generado el concepto de capital social, muchas veces ligado a teorías de la acción totalmente opuestas, las cuales se han sistematizado y discutido en otra parte (Gutiérrez, 2005 y 2008), se señala una dimensión que se considera fundamental.

Por un lado, hay una conceptualización (probablemente la más extendida) que se sustenta en una visión normativa y en cierto modo, voluntarista e ingenua, la cual sostiene que el capital social constituye una suerte de modelo ideal de organización social, de una especie de comunidad en la cual sus miembros deben actuar solidariamente, "con el fin de aumentar su resiliencia y su capacidad colectiva de emprender proyectos" (Durston, 2002: 44). Esa mirada, con matices, analiza la vida social en términos consensuales, centrando la atención en la sinergia que se desarrolla entre la institucionalidad del capital social y el Estado (Evans, 1996 y Putnam, 2000), en los compromisos personales de los funcionarios con la comunidad local (Tendler, 1997), en la confianza de los miembros de una sociedad (Fukuyama, 2000) o en la confianza, la reciprocidad y la cooperación como elementos constitutivos del capital social (Putnam, op. cit.).

Es fundamental tomar una conceptualización del capital social (Bourdieu, 1980) que asuma los rasgos centrales de nuestro mundo social: estructurado en relaciones de clases, cuya reproducción (y con ellas las de los mecanismos de dominación-dependencia) depende de la reproducción de estructuras objetivas y de agentes dotados de diferentes tipos de recursos que luchan por mantener o mejorar su posición en esas relaciones. Como el capital en Marx, el capital social aquí no es una cosa, sino una relación, que constituye una fuente de poder y de conflicto, en el marco de una estructura de fuerzas y de luchas.

Apartar las conceptualizaciones ingenuas, supone tomar la realidad social como se presenta y asumir el desafío de consolidar formas asociativas $y$ procesos autogestivos, a partir de análisis realistas. Es decir, considerar las relaciones de conflicto $y$ de poder, tanto en el marco del espacio social global como en el seno mismo de esos espacios de "capital social colectivo" (Gutiérrez, 2004), constituye el único 
camino para generar estrategias que permitan, sino eliminarlos, al menos contrarrestarlos todo lo que fuese posible.

Por otra parte, en consonancia con todo lo planteado, es de fundamental importancia, en el marco de una sociedad donde los recursos están desigualmente repartidos, sostener las diferentes propuestas a partir de análisis claros acerca de esos recursos, tanto en el plano material-objetivo, como en el sentido de capital incorporado. Es decir, recordar que la vida social existe como condiciones objetivas externas y como condiciones objetivas incorporadas (Bourdieu, 1979), como disposiciones a actuar, a percibir, a visualizar como posible, lo que ha venido siendo viable a lo largo de trayectorias individuales y colectivas. De este modo, un cambio de sentido positivo en el marco de las condiciones materiales de existencia necesita estar acompañado de disposiciones que permitan valorarlo como tal y que estén en condiciones de generar prácticas que permitan sostenerlo.

Es aquí donde la política y los programas concretos pueden incidir, teniendo un conocimiento claro de la realidad territorial en la que se asientan. Esta dimensión territorial-local es fundamental para analizar las relaciones concretas que estructuran los diversos tejidos sociales, una vez que se han tomado las decisiones teórico-políticas fundamentales $y$ a fin de sustentar las político-instrumentales.

\section{BIBLIOGRAFÍA}

Auyero, J. La politica de los pobres. Las prácticas clientelísticas del peronismo. Argentina: Editorial Manantial, 2001.

Auyero, J. "Los cambios en el repertorio de la protesta social en Argentina". Desarrollo Económico 42 (166). Julio-septiembre 2002: $187-210$.

Auyero, J. La protesta. Retratos de la beligerancia popular en la Argentina democrática. Argentina: Editorial Libros del Rojas, 2002.

Barbeito, A. y Lo Vuolo, R. La modernización excluyente. Transformación económica y Estado de Bienestar en Argentina. Argentina: Editorial UNICEF-CIEPPLosada, 1995.
Beccaria, L. "Estancamiento y distribución del ingreso". Desigualdad y exclusión. Desafíos para la política social en la Argentina de fin de siglo. Minujin, A. (ed.). Argentina: Editorial UNICEF-Losada, 1993.

Beccaria, L. "Dimensiones y alcances de la crisis argentina". Material de cátedra Economía I. FLACSO, 2006.

Benza, G. y Calvi, G. "Reestructuración económica, concentración del ingreso y ciclos de desigualdad (1974-2003)". Realidad Económica 214. IADE, 2004: 74-104.

Bourdieu, P. La distinction. Francia: Editorial Minuit, 1979.

Bourdieu, P. "Le capital social. Notes provisoires". Actes de la Recherche en Sciences Sociales 31. Éditions du Seuil, 1980: 2-3.

Bustelo, E. Política social en un contexto de crisis: ¿será que se puede? Argentina: Editorial UNICEF-Losada, 1988.

Bustelo, E. (1992). "La producción del Estado de Malestar”. Cuesta abajo. Los nuevos pobres: efectos de la crisis en la sociedad argentina. En: Minujin, A. et ál. $2 \mathrm{da}$. edición. Argentina: Editotial UNICEFLosada, 1993.

Castel, R. (1977). La metamorfosis de la cuestión social. Una crónica del salariado. 2 da. edición. Argentina: Editorial Paidós, 1997.

Cortés, R.; Groisman, F. y Hoszowski, A. "Transiciones ocupacionales: el caso del plan jefes y jefas". Realidad Económica 202. 2004: 11-28.

Durston, J. El capital social campesino en la gestión del desarrollo rural. Díadas, equipos, puentes y escaleras. Chile: Editorial CEPAL, 2002.

Evans, P. State-society synergy. Government and social capital in development. Estados Unidos: International and Area Studies, 1996.

Fukuyama, F. A grande ruptura. Brasil: Editorial Rocco, 2000.

Esquivel, V. y Maurizio, R. "La desigualdad de los ingresos $y$ otras inequidades en Argentina post-convertibilidad". Policy 
Paper Series Workshop. Economics Working Group (EWG), 2005.

Giacometti, C. y Monza, A. "Los beneficiarios del Plan Jefes y Jefas de Hogar. Enfrentando los retos al trabajo decente en la crisis argentina". Proyecto de Cooperación Técnica OIT/ Gobierno argentino (MTESS). Serie de Documentos de Trabajo 1. Agosto, 2003.

Grassi, E.; Hintze, S. y Neufeld, M. R. Politicas sociales, crisis y ajuste estructural. Argentina: Espacios Editorial, 1994.

Grassi, E. Políticas y problemas sociales en la sociedad neoliberal. La otra década infame I. Argentina: Espacios Editorial, 2003a.

Grassi, E. "Política, cultura y sociedad: la experiencia neoliberal en la Argentina". Entre el trabajo y la politica. Las reformas de las politicas sociales argentinas en perspectiva comparada. Lindenboim, J. y Danani, C. (coords.). Argentina: Editorial Biblos, 2003b.

Grassi, E. Politica y cultura en la sociedad neoliberal. La otra década infame II. Argentina: Espacios Editorial, 2004.

Guimenez, S. y Zibechi, C. "El sentido del trabajo y la subjetividad de los 'beneficiarios'. Desafíos para futuras orientaciones en materia de políticas sociales". Documento presentado en el $7^{\circ}$ Congreso Nacional de Estudios del Trabajo. ASET, 2005.

Gutiérrez, A. Pobre como siempre... Estrategias de reproducción social en la pobreza. Argentina: Editorial Ferreyra Editor, 2004.

Gutiérrez, A. "Acerca de la noción de capital social como herramienta de análisis. Reflexiones teóricas en torno a un caso empírico". Perspectivas. Sobre la administración, las politicas públicas y el Estado 2. Universidad Nacional del Comahue, 2005: 7-26.

Gutiérrez, A. "El 'capital social' en la pobreza: apuesta, medio y resultado de luchas simbólicas". Estudios sobre pobreza en Argentina. Aproximaciones teórico- metodológicas. Pavcovich, P. y Truccone, D. (comps.). Argentina: Editorial de la Universidad Nacional de Villa María, 2008.

Hintze, S. "Exclusión, derechos y políticas sociales. La promoción de formas asociativas $y$ trabajo autogestivo en la Argentina”. Fermentum 45. Enero-abril 2006: 100-137.

Lodola, G. "Protesta popular y redes clientelares en la Argentina: el reparto federal del Plan Trabajar (1996-2001)". Desarrollo Económico 44 (176). Eneromarzo 2005: 515-536.

Lo Vuolo, R. "La economía política del ingreso ciudadano". Contra la exclusión. La propuesta del ingreso ciudadano. Lo Vuolo, Rubén (comp.). Argentina: Editorial Miño Dávila-CIEPP, 1995.

Minujin, A. (1992). "Prólogo". Cuesta abajo. Los nuevos pobres: efectos de la crisis en la sociedad argentina. Minujin, A. et ál. (eds.). 2da edición. Argentina: Editorial UNICEF-Losada, 1993.

Minujin, A. "Introducción". Desigualdad y exclusión. Desafíos para la política social en la Argentina de fin de siglo. Minujin, A. (ed.). Argentina: Editorial UNICEFLosada, 1993: 9-25.

Murmis, M. y Feldman, S. (1992). "La heterogeneidad social de las pobrezas". Cuesta abajo. Los nuevos pobres: efectos de la crisis en la sociedad argentina. Minujin, A. et ál. (eds.). 2da. Edición. Argentina: Editorial UNICEF-Losada, 1993.

Pautassi, L. "Beneficios y beneficiarias: análisis del programa jefes y jefas de hogar desocupados de Argentina". Politicas de empleo para superar la pobreza. Chile. Editorial: OIT, 2003.

Putnam, R. Comunidade e democracia: a experiência da Itália Moderna. Brasil: Editorial Editora FGV, 2000.

Rosanvallon, P. La nueva cuestión social. Repensar el Estado Providencia. Argentina: Editorial Manantial, 1995.

Svampa, M. La sociedad excluyente: La Argentina bajo el signo del 
neoliberalismo. Argentina: Editorial Taurus, 2005.

Svampa, M. y Pereyra, S. Entre la ruta y el barrio. Argentina: Editorial Paidós, 2003.

Tecco, C. y Bressan, J. "Área Metropolitana Córdoba: análisis de asentamientos y de sus articulaciones al sistema urbano metropolitano. Los casos Estación General Paz y Malvinas Argentinas”.
Informe de investigación 2000-2003. Universidad Nacional de Córdoba, 2003.

Tendler, J. Good government in the tropics. Estados Unidos: Editorial Johns Hopkins University Press, 1997.

Fecha ingreso: 29/11/2011 Fecha de aprobación: 10/02/2012 
